\title{
Satisfação e percepção de dor em puérperas: um estudo comparativo após parto vaginal $e$ cesariana em maternidades públicas de Aracaju
}

\author{
Pain perception and satisfaction of postpartum women: a comparative \\ study after vaginal and caesarean birth in Aracaju public hospitals
}

\author{
Mariana Tirolli Rett', Danieli Moreira de Oliveira², Ellen Caroline Gabriel Soares', \\ Josimari Melo DeSantana ${ }^{1,3}$, Karina Conceição Gomes Machado de Araújo4 \\ ${ }^{1}$ Departamento de Fisioterapia, Universidade Federal de Sergipe (UFS) - São Cristóvão (SE), Brasil. \\ Instituto Federal de Sergipe (IFS) - Aracajú (SE), Brasil. \\ 3Programa de Pós-Graduação em Ciências Fisiológicas e em Ciências da Saúde, UFS - São Cristóvão (SE), Brasil. \\ ${ }^{4}$ Departamento de Morfologia do Programa de Pós-Graduação em Ciências Fisiológicas e em Ciências da Saúde, UFS - São Cristóvão (SE), Brasil.
}

DOI: http://dx.doi.org/10.7322/abcshs.v42i2.1005

\begin{abstract}
RESUMO
Introdução: Além da qualidade do cuidado, estrutura física e acesso ao serviço de saúde, a assistência humanizada no processo de nascimento deve refletir sobre a experiência de dor e satisfação da mulher. Objetivo: Comparar a satisfação e a percepção de dor vivenciada pela mulher no parto vaginal e na cesariana. Métodos: Estudo descritivo de corte transversal envolvendo puérperas. Foram aplicados: "Questionário de Experiência e Satisfação com o Parto" (QESP) modificado, versão curta do "questionário de dor McGill" e "esquema de representação corporal feminino". Resultados: Foram entrevistadas 150 puérperas. Após a cesariana, demonstraram maior satisfação quanto à forma que decorreu o trabalho de parto (TP) $(p=0,01)$ e menos satisfação em relação à dor no pós-parto $(P P)(p=0,04)$. As de parto vaginal estavam mais satisfeitas sobre como decorreu o PP $(p=0,02)$ e menos satisfeitas com a intensidade de dor no TP $(p=0,03)$ e no parto $(P)(p=0,01)$. As características dolorosas durante o TP e $P$ foram aguda e cruel-punitiva. Em relação ao PP, a dor em baixo ventre foi mais relatada após a cesariana. A intensidade dolorosa no TP foi significativamente maior no parto vaginal $(7,30( \pm 2,82)$ versus 5,86 ( $\pm 3,51),(p=0,007)$. Conclusão: As que realizaram cesariana estavam mais satisfeitas como decorreu o TP e menos satisfeitas em relação à dor no PP. As de parto vaginal estavam mais satisfeitas com a forma que decorreu o PP e menos satisfeitas com a intensidade de dor no TP e P.
\end{abstract}

Palavras-chave: período pós-parto; dor do parto; satisfação do paciente; trabalho de parto; parto normal; cesárea.

\section{ABSTRACT}

Introduction: In addition to the quality of care, physical structure and access to health services, a humanized care during birth process should reflect on the women's experience of pain and satisfaction. Objective: To compare the satisfaction and perception of pain experienced by women during vaginal and cesarean delivery processes. Methods: Descriptive cross-sectional study involving women in the immediate postpartum period. The modified "Experience and Satisfaction with Childbirth Questionnaire" ( "Questionário de Experiência e Satisfação com o Parto"-QESP), the short version of "McGill pain questionnaire" and the "female body representation scheme" were used. Results: 150 postpartum women were interviewed. After cesarean section demonstrated more satisfaction regarding the way the labor (LB) was carried out $(p=0.01)$ and less satisfaction with pain in the postpartum period (PP) $(p=0.04)$. Those submitted to vaginal delivery were more satisfied with their PP $(p=0.02)$ and less satisfied with the intensity of pain during $L B(p=0.03)$ and at birth $(B)(p=0.01)$. The pain described during LB and B were "acute" and "cruel-punitive". In relation to $\mathrm{PP}$, pain in the lower belly was more often reported after cesarean sections. Pain intensity during LB was significantly higher in vaginal labor $(7,30( \pm 2,82)$ versus $5,86( \pm 3,51),(p=0,007)$. Conclusion: The perform cesarean sections were more satisfied with the way LB was carried out and less satisfied in relation to pain in the PP. Women who underwent vaginal labor were more satisfied with the PP and less satisfied with the intensity of pain during LB and at B.

Keywords: postpartum period; labor pain; patient satisfaction; labor, obstetric; natural childbirth; cesarian section.

Recebido em: 13/05/2016

Revisado em: 06/12/2016

Aprovado em: 24/02/2017

Autor para correspondência: Mariana Tirolli Rett - Departamento de Fisioterapia da Universidade Federal de Sergipe (UFS) - Avenida Marechal Rondon, s/n Jardim Rosa Elze - CEP: 49100-000 - São Cristóvão (SE), Brasil - E-mail: marianatrb@gmail.com

Conflito de interesses: nada a declarar.

Fonte de financiamento: Programa Institucional de Bolsas de Iniciação à Extensão (PIBIX) - Universidade Federal de Sergipe 


\section{INTRODUÇÃO}

A assistência ao parto tem uma trajetória histórica que acompanhou diversas mudanças sociopolíticas e culturais do Brasil e representa um tema de grandes reflexões ao longo de várias décadas. O período gestacional é peculiar na vida de uma mulher, portanto, merece ser conduzido de forma adequada por profissionais qualificados e por maternidades estruturadas. A partir de conceitos sobre direitos reprodutivos, saúde materna e perinatal, criaram-se novos modelos de atenção, nos quais a percepção da mulher como sujeito, o respeito aos direitos reprodutivos e a perspectiva da humanização da assistência aparecem como elementos norteadores ${ }^{1-5}$.

OProgramadeHumanizaçãono Pré-nataleNascimento(PHPN) propõe estratégias para a humanização do atendimento, facilitar o acesso ao serviço de saúde às gestantes, oferecer qualidade no acompanhamento durante o pré-natal ( $\mathrm{PN})$, assistência ao parto, puerpério e mãe-bebê $\hat{}^{1}$, pois entende que a mulher deve ser participante ativa do processo de parto. Além da estrutura física, da qualidade do cuidado, do acesso aos serviços, da postura dos profissionais e dos usuários, a assistência humanizada deve valorizar a escuta e refletir sobre a satisfação da mulher com o processo do nascimento, envolvendo trabalho de parto, parto e puerpério, além da percepção de dor vivenciada neste contexto ${ }^{2-5}$.

Nacionalmente, já existem estudos que mostram a relação entre a satisfação das mulheres e a equipe de assistência, presença de acompanhantes, tempo decorrido até o primeiro contato com o recém-nascido, técnicas de manejo da dor e tipo de parto ${ }^{6-10}$. Mas, ainda, a intensidade da dor destaca-se como fator que pode prejudicar a satisfação e assim, gerar insatisfação no puerpério para muitas mães ${ }^{4,10-14}$. Entende-se que a dor do parto tem uma dimensão sensorial e outra afetiva, cuja interpretação é influenciada por uma variedade de fatores emocionais, socioculturais e cognitivos ${ }^{4,7,8,15}$.

Em maternidades públicas da região nordeste, poucas investigações foram conduzidas na área de saúde materno-infantil ${ }^{16}$, principalmente com relação à temática de satisfação da parturiente/puérpera e percepção de dor durante os trabalho de parto (TP), parto (P) e pós-parto (PP). Portanto, conhecer a percepção e características da dor vivenciadas por estas mulheres durante este período poderá contribuir com o processo de humanização da assistência ao parto e auxiliar na escolha do melhor método de manejo da dor. O objetivo deste estudo foi avaliar e comparar a satisfação com o processo de nascimento, envolvendo o trabalho de parto, parto e pós-parto, além da percepção de dor vivenciada pela mulher após o parto vaginal e cesariana.

\section{MÉTODOS}

Foi conduzido um estudo descritivo de corte transversal incluindo puérperas primíparas e multíparas, que realizaram parto vaginal (espontâneo ou induzido) ou à cesárea não eletiva, idade gestacional a termo, respeitando-se um mínimo de 8 horas após o parto e máximo de 16 horas. Foram excluídas aquelas que tiveram natimorto, após curetagem ou idade inferior a 18 anos.
Os dados foram coletados em duas maternidades públicas do município de Aracaju/SE e o contato com as mulheres foi apenas no pós-parto (puerpério), sendo no alojamento conjunto das enfermarias. Foi realizada uma entrevista utilizando quatro instrumentos. Um formulário que abordou aspectos sociodemográficos, antecedentes obstétricos e gestação atual, composto de 21 itens elaborados pelos pesquisadores. Um questionário que avaliou a satisfação da paciente, considerando 16 itens exclusivamente relacionados a essa variável, pertencentes ao Questionário de Experiência e Satisfação com o Parto (QESP) $)^{2}$ modificado, o qual investiga a satisfação da mulher relacionada à forma e ao tempo dos TP, parto e PP, condições físicas da maternidade, qualidade dos cuidados prestados, intensidade da dor e tempo para pegar no bebê. Este questionário não apresenta escore e sim as respostas "nada", "um pouco", "bastante" ou "muito" satisfeita. Outro questionário que avaliou a percepção de dor, correspondente à versão curta do questionário de dor McGill ${ }^{17}$, o qual caracteriza a dor a partir de descritores e a intensifica por meio da escala analógica visual (EAV, utilizada para o TP) e do índice de intensidade da dor atual (IDA, utilizado para o PP). Ao final, também para a avaliação da variável dor, aplicou-se um esquema de representação corporal feminino para localização de pontos dolorosos ocorridos durante o TP e PP, também elaborado pelos pesquisadores. As informações sobre o TP e parto foram obtidas pelo método de coleta recordatório. O projeto foi aprovado pelo Comitê de Ética em Pesquisa da Universidade Federal de Sergipe (UFS) (CAAE: 04674712.2.0000.0058) e todas assinaram o Termo de Consentimento Livre e Esclarecido. Apenas uma pesquisadora realizou a coleta de dados.

Para o cálculo amostral considerou-se uma média de 400 partos por mês para uma das maternidades, que é de alta complexidade, e uma média de 1.200 partos por mês para a outra maternidade, de baixo risco. Considerando-se 6.400 partos durante os 4 meses de pesquisa (julho a outubro de 2012), estimando-se uma frequência de $50 \%$ e um erro aceitável de $40 \%$, foi calculado tamanho amostral de 150 mulheres com um nível de 95\% de confiança. Foi utilizado o software EPIINFO 7.

Foram utilizadas médias e desvio-padrão, frequência e porcentagem. Para comparação de médias foi utilizado o teste $t$ de Student e para comparação de proporções o teste $\chi^{2}$. Os dados foram analisados pelo BioEstat 5.0, considerando o nível de significância de $\mathrm{p}<0,05$.

\section{RESULTADOS}

Foram selecionadas 154 puérperas, duas se recusaram a assinar o TCLE e duas foram excluídas por dados incompletos, assim, foram incluídas 150 puérperas.

A maioria das puérperas era jovem, do lar, da cor parda, estudaram até o ensino médio incompleto, tinham união estável, residiam no interior do Estado e com baixa renda familiar (Tabela 1). Quanto maior o número de moradores na mesma casa 
(5 ou mais), menor a renda familiar abaixo de 3 salários mínimos $(\mathrm{p}=0,001)$. Para chegar à maternidade, a minoria foi encaminhada de uma Unidade Básica de Saúde (UBS) e a maioria utilizou carro próprio (Tabela 1).

A maioria afirmou não ter planejado a gravidez, mas realizou o PN, desde o primeiro trimestre e com mais de 6 consultas. $\mathrm{Na}$ assistência $\mathrm{PN}$, consideraram-se orientadas em relação às alterações da gravidez, não orientadas em relação ao TP e PP, assim como não participaram de atividades em grupo. Na ocasião da entrevista, 95 (63,3\%) encontravam-se no PP vaginal, sendo a maioria multípara. Entre as primíparas, a via de parto foi em $56,9 \%$ dos casos vaginal e em $43,1 \%$ cesárea. Enquanto entre as multíparas, $58,8 \%$ haviam realizado exclusivamente partos vaginais, $24,7 \%$ a partos vaginais e a cesarianas e $16,5 \%$ apenas a cesarianas (Tabela 2).

Quanto à experiência e satisfação, as que realizaram cesariana demonstraram estar mais satisfeitas em relação à forma como decorreu o $\mathrm{TP}(\mathrm{p}=0,01)$, ao tempo que demorou o parto $(\mathrm{p}=0,03)$ e à qualidade dos cuidados prestados no parto $(\mathrm{p}=0,01)$. Enquanto as que realizaram parto vaginal estavam mais satisfeitas com a forma como decorreu o PP ( $\mathrm{p}=0,02)$ e menos satisfeitas com a intensidade de dor sentida no TP $(\mathrm{p}=0,03)$ e no parto $(\mathrm{p}=0,01)$, ao contrário

Tabela 1: Características pessoais, sociais e demográficas das puérperas de maternidades públicas de Aracaju (SE) $(n=150)$

\begin{tabular}{|l|c|c|}
\hline Características pessoais, sociais e demográficas \\
\hline Idade & $\mathbf{2 6 , 0}$ & $\pm \mathbf{6 , 0}$ \\
\hline Anos de estudo & $\mathbf{n}$ & $\%$ \\
\hline$\quad$ Nenhum & 1 & 0,7 \\
\hline 1 a 4 & 13 & 8,7 \\
\hline 5 a 8 & 52 & 34,7 \\
\hline 9 a 11 & 74 & 49,3 \\
\hline 12 e mais & 10 & 6,7 \\
\hline Mora com o companheiro & & \\
\hline Sim & 127 & 84,7 \\
\hline Não & 23 & 15,3 \\
\hline Residência & & \\
\hline Capital (Aracaju) & 71 & 47,3 \\
\hline Interior do Estado & 73 & 48,7 \\
\hline Outros Estados & 6 & 4,0 \\
\hline Procedência & & \\
\hline Casa & 92 & 61,3 \\
\hline UBS & 9 & 6,0 \\
\hline Hospital Especializado & 49 & 32,7 \\
\hline Transporte até a maternidade & & \\
\hline Carro próprio & 82 & 54,7 \\
\hline Transporte coletivo & 11 & 7,3 \\
\hline Ambulância & 57 & 38,0 \\
\hline Número de moradores em casa & & \\
\hline 1 a 2 & 20 & 13,3 \\
\hline 3 a 4 & 74 & 49,3 \\
\hline 5 e mais & 56 & 37,4 \\
\hline Renda familiar (salário-mínimo) & 52 & 34,7 \\
\hline$\leq 1$ & 85 & 56,7 \\
\hline$>1$ e $\leq 2$ & 13 & 8,6 \\
\hline 3 ou mais & & \\
\hline & & \\
\hline
\end{tabular}

das que realizaram cesariana, que apresentaram menor satisfação em relação à dor sentida no $\mathrm{PP}(\mathrm{p}=0,04)$. Para as outras variáveis de satisfação não houve diferença significativa (Tabela 3).

Quanto à percepção de dor, a dor foi caracterizada durante o TP e o parto, como dor aguda especialmente no parto vaginal e, dolorida e em cólica em ambos os grupos (Figura 1). Quanto à localização, a queixa mais comum foi dor no baixo ventre e lombossacra as puérperas de parto vaginal e no baixo ventre, lombossacra e abdômen naquelas que realizaram cesariana (Figura 2).

Em relação ao PP, as dores no baixo ventre e no abdômen foram mais relatadas após a cesariana e a dor na vagina foi mais relatada após o parto vaginal.

Quando comparada a dor pela EAV no TP, as puérperas de parto vaginal apresentaram média de $7,30( \pm 2,82)$, e as de cesariana $5,86( \pm 3,51)$, sendo significativamente maior $(\mathrm{p}=0,007)$ no parto vaginal. Para a IDA no PP, a maior parte das puérperas de parto

Tabela 2: Distribuição absoluta e percentual dos dados da gestação e parto de puérperas em maternidades públicas de Aracaju (SE) $(\mathrm{n}=150)$

\begin{tabular}{|c|c|c|}
\hline Dados obstétricos & $\mathbf{n}$ & $\%$ \\
\hline Gravidez planejada & 48 & 32,0 \\
\hline Pré-natal & 147 & 98,0 \\
\hline \multicolumn{3}{|l|}{ Início do pré-natal (trimestre) } \\
\hline $1^{\circ}$ & 113 & 75,3 \\
\hline $2^{\circ}$ & 31 & 20,7 \\
\hline $3^{\circ}$ & 6 & 4,0 \\
\hline \multicolumn{3}{|l|}{ Número de consultas pré-natal } \\
\hline 1 a 3 & 19 & 12,7 \\
\hline 4 a 6 & 63 & 42,0 \\
\hline Mais de 6 & 68 & 45,3 \\
\hline $\begin{array}{l}\text { Orientações no pré-natal quanto a } \\
\text { alterações da gravidez }\end{array}$ & 87 & 58,0 \\
\hline $\begin{array}{l}\text { Orientações no pré-natal quanto ao } \\
\text { trabalho de parto }\end{array}$ & 52 & 34,7 \\
\hline $\begin{array}{l}\text { Orientações no pré-natal quanto ao } \\
\text { pós-parto }\end{array}$ & 41 & 27,3 \\
\hline $\begin{array}{l}\text { Participação no pré-natal de atividades } \\
\text { em grupo de gestantes }\end{array}$ & 38 & 25,3 \\
\hline \multicolumn{3}{|l|}{ Avaliação da assistência pré-natal } \\
\hline Boa & 106 & 70,7 \\
\hline Regular & 32 & 21,3 \\
\hline Ruim & 12 & 8,0 \\
\hline \multicolumn{3}{|l|}{ Idade gestacional (semanas) } \\
\hline$\leq 37$ & 16 & 10,7 \\
\hline$>37$ & 134 & 89,3 \\
\hline \multicolumn{3}{|l|}{ Paridade } \\
\hline Primíparas & 65 & 56,7 \\
\hline Multíparas & 85 & 43,3 \\
\hline \multicolumn{3}{|l|}{ Tipo de parto (atual) } \\
\hline Vaginal & 95 & 63,3 \\
\hline Cesáreo & 55 & 36,7 \\
\hline \multicolumn{3}{|l|}{ Tempo de trabalho de parto vaginal (horas) } \\
\hline$\leq 5$ & 52 & 54,7 \\
\hline$>5$ & 43 & 45,3 \\
\hline \multicolumn{3}{|l|}{ Tempo de trabalho de parto cesárea (horas) } \\
\hline$\leq 5$ & 20 & 35,5 \\
\hline$>5$ & 35 & 64,5 \\
\hline
\end{tabular}


Tabela 3: Distribuição absoluta e percentual da satisfação de puérperas após parto vaginal e cesariana em relação ao trabalho de parto $(\mathrm{TP})$, parto $(\mathrm{P})$ e pós-parto (PP)

\begin{tabular}{|c|c|c|c|c|c|c|c|}
\hline \multirow{3}{*}{$\begin{array}{l}\text { Satisfação relacionada ao Trabalho de } \\
\text { parto (TP), parto (P) e pós-parto (PP) / } \\
\text { parto vaginal e cesárea. }\end{array}$} & \multicolumn{3}{|c|}{ Vaginal $(n=95)$} & \multicolumn{3}{|c|}{ Cesárea $(n=55)$} & \multirow{3}{*}{ Valor $p$} \\
\hline & Nada & Pouco & Muito & Nada & Pouco & Muito & \\
\hline & $n(\%)$ & $n(\%)$ & $n(\%)$ & $\mathrm{n}(\%)$ & $n(\%)$ & $n(\%)$ & \\
\hline \multicolumn{8}{|l|}{ Forma como decorreu o } \\
\hline TP & $18(18,9)$ & $36(37,9)$ & $41(43,2)$ & $6(10,9)$ & $13(23,6)$ & $36(65,5)$ & $0,01^{*}$ \\
\hline $\mathrm{P}$ & $7(7,4)$ & $24(25,3)$ & $64(67,4)$ & $3(5,5)$ & $9(16,4)$ & $43(78,2)$ & 0,36 \\
\hline PP & $4(4,2)$ & $24(25,3)$ & $67(70,5)$ & $9(16,4)$ & $15(27,3)$ & $31(56,4)$ & $0,02^{*}$ \\
\hline \multicolumn{8}{|l|}{ Tempo que demorou o } \\
\hline TP & $18(18,9)$ & $35(36,8)$ & $42(44,2)$ & $13(23,6)$ & $14(25,5)$ & $28(50,9)$ & 0,35 \\
\hline$P$ & $9(9,5)$ & $23(24,2)$ & $63(66,3)$ & $3(5,5)$ & $5(9,1)$ & $47(85,5)$ & $0,03^{*}$ \\
\hline PP & $8(8,4)$ & $47(49,5)$ & $40(42,1)$ & $6(10,9)$ & $23(41,8)$ & $26(47,3)$ & 0,66 \\
\hline \multicolumn{8}{|l|}{ Condições físicas da maternidade no } \\
\hline TP & $15(15,8)$ & $21(22,1)$ & $59(62,1)$ & $7(12,7)$ & $20(36,4)$ & $28(50,9)$ & 0,16 \\
\hline $\mathrm{P}$ & $7(7,4)$ & $28(29,5)$ & $60(63,2)$ & $4(7,3)$ & $11(20,0)$ & $40(72,7)$ & 0,431 \\
\hline PP & $4(4,2)$ & $20(21,1)$ & $71(74,7)$ & $4(7,3)$ & $14(25,5)$ & $37(67,3)$ & 0,554 \\
\hline \multicolumn{8}{|l|}{ Qualidade dos cuidados prestados no } \\
\hline TP & $12(12,6)$ & $25(26,3)$ & $58(61,1)$ & $3(5,5)$ & $14(25,5)$ & $38(69,1)$ & 0,34 \\
\hline $\mathrm{P}$ & $5(5,3)$ & $22(23,2)$ & $68(71,6)$ & $0(0)$ & $5(9,1)$ & $50(90,9)$ & 0,01 \\
\hline PP & $5(5,3)$ & $24(25,3)$ & $66(69,5)$ & $4(7,3)$ & $12(21,8)$ & $39(70,9)$ & 0,81 \\
\hline \multicolumn{8}{|l|}{ Intensidade de dor que sentiu no } \\
\hline TP & $45(47,4)$ & $25(26,3)$ & $25(26,3)$ & $19(34,5)$ & $10(18,2)$ & $26(47,3)$ & 0,03 \\
\hline $\mathrm{P}$ & $30(31,6)$ & $26(27,4)$ & $39(41,1)$ & $5(9,1)$ & $8(14,5)$ & $42(76,4)$ & 0,01 \\
\hline PP & $8(8,4)$ & $28(29,5)$ & $59(62,1)$ & $11(20,0)$ & $19(34,5)$ & $25(45,5)$ & 0,04 \\
\hline Tempo que levou para pegar no seu bebê & $10(10,5)$ & $16(16,8)$ & $69(72,6)$ & $9(16,4)$ & $12(21,8)$ & $34(61,8)$ & 0,36 \\
\hline
\end{tabular}

${ }^{*} \mathrm{p}<0,05$ Teste do $\chi^{2}$

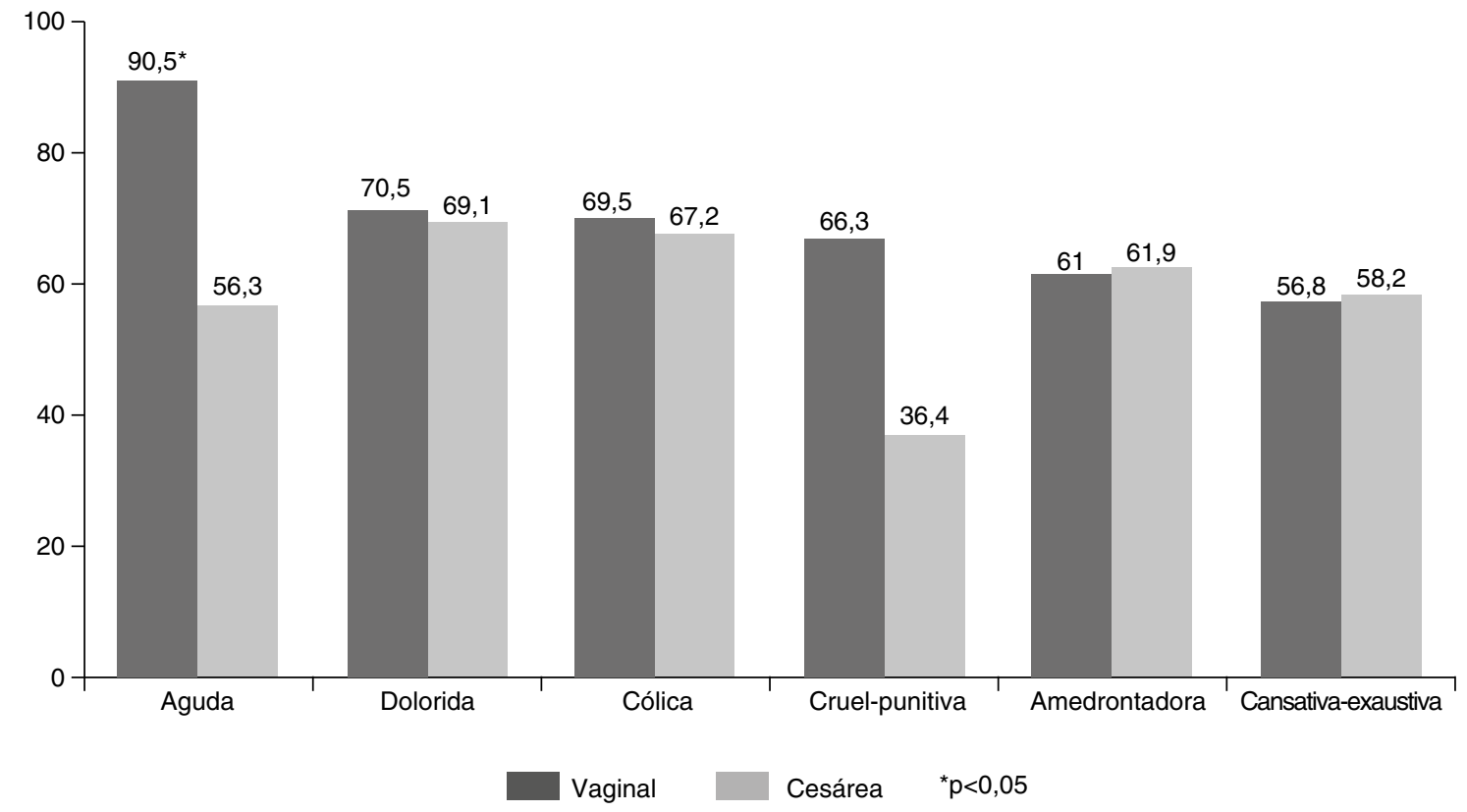

Figura 1: Caracterização da dor durante o trabalho de parto vaginal $(n=95)$ e cesariana $(n=55)$ 
vaginal optou pela resposta " $0=$ sem dor", já as de cesariana relataram dor " $2=$ desconfortante".

\section{DISCUSSÃO}

A maior parte da amostra foi composta por mulheres jovens, de baixa renda familiar, com ensino médio incompleto e residentes no interior do estado. Percebe-se que a maioria das que utiliza as maternidades vinculadas ao Sistema Único de Saúde (SUS) no município de Aracaju, são aquelas com menor poder aquisitivo, contrariando a proposta de universalidade do SUS. O mesmo pode ser observado por meio de estudo epidemiológico-social de saúde materno-infantil realizado em Sergipe ${ }^{16}$, onde as maiores proporções de partos ocorreram na faixa etária de 20 a 24 anos, a renda familiar abaixo de 2 salários mínimos, baixa escolaridade e o predomínio da escolaridade de 9 a 11 anos de estudo. Destaca-se também, a grande demanda de moradoras de outros municípios, o que pode ser um fator contribuinte para a sobrecarga dos serviços nas maternidades públicas da capital.

Quanto ao PN, quase todas realizaram o mínimo de 6 consultas e, apesar de se considerarem pouco orientadas em relação ao processo do parto e sem participar de atividades coletivas, a maior parte delas avaliou bem o PN. Existem regiões no nordeste do Brasil em que o PN ainda é inadequado, mas observa-se que sua importância é bem reconhecida ${ }^{9,18}$. Provavelmente, esta inadequação pode fazer com que estas mulheres pensem que apenas as consultas e exames laboratoriais fazem parte do PN e, portanto, mesmo não tendo sido orientadas, acreditam que o objetivo do PN foi cumprido.

Em um aspecto geral, demonstraram-se satisfeitas com a estrutura física das maternidades, com os cuidados prestados pelos profissionais de saúde e com o tempo que levou para pegar o bebê. Independentemente da via de parto, grande parte não estava satisfeita com o tempo que durou o PP, pois a demora no tempo de internação após o parto geralmente é acompanhada pela ansiedade de voltar para casa junto à família e pelo medo de complicações com a saúde do bebê. Destaca-se que grande parte da insatisfação esteve relacionada à intensidade da dor durante o TP e parto no parto vaginal e no PP na cesariana, denotando que independente da via de parto a dor esteve presente em diferentes momentos do processo. Estudos anteriores identificaram que de forma geral, as mulheres mostraram-se insatisfeitas com a intensidade de dor sentida durante o TP $(72,1 \%)^{15}$ e no $\mathrm{PP}^{10}$.

Embora a intensidade de dor no TP do parto vaginal tenha sido significativamente maior $(\mathrm{p}=0,007)$, a dor sentida no TP foi caracterizada de forma semelhante pelas puérperas de parto vaginal e cesariana, tanto quanto à sua descrição como à localização dos pontos dolorosos. Como no presente estudo, muitas puérperas que realizaram cesariana estiveram em TP por mais de 5 horas e as mesmas compartilharam uma vivência próxima àquelas de parto vaginal. Vale lembrar que em algumas situações, mesmo com gestação de baixo risco, pode haver falha na evolução do TP e a via final ser a cesariana. Como não foram investigados os motivos da

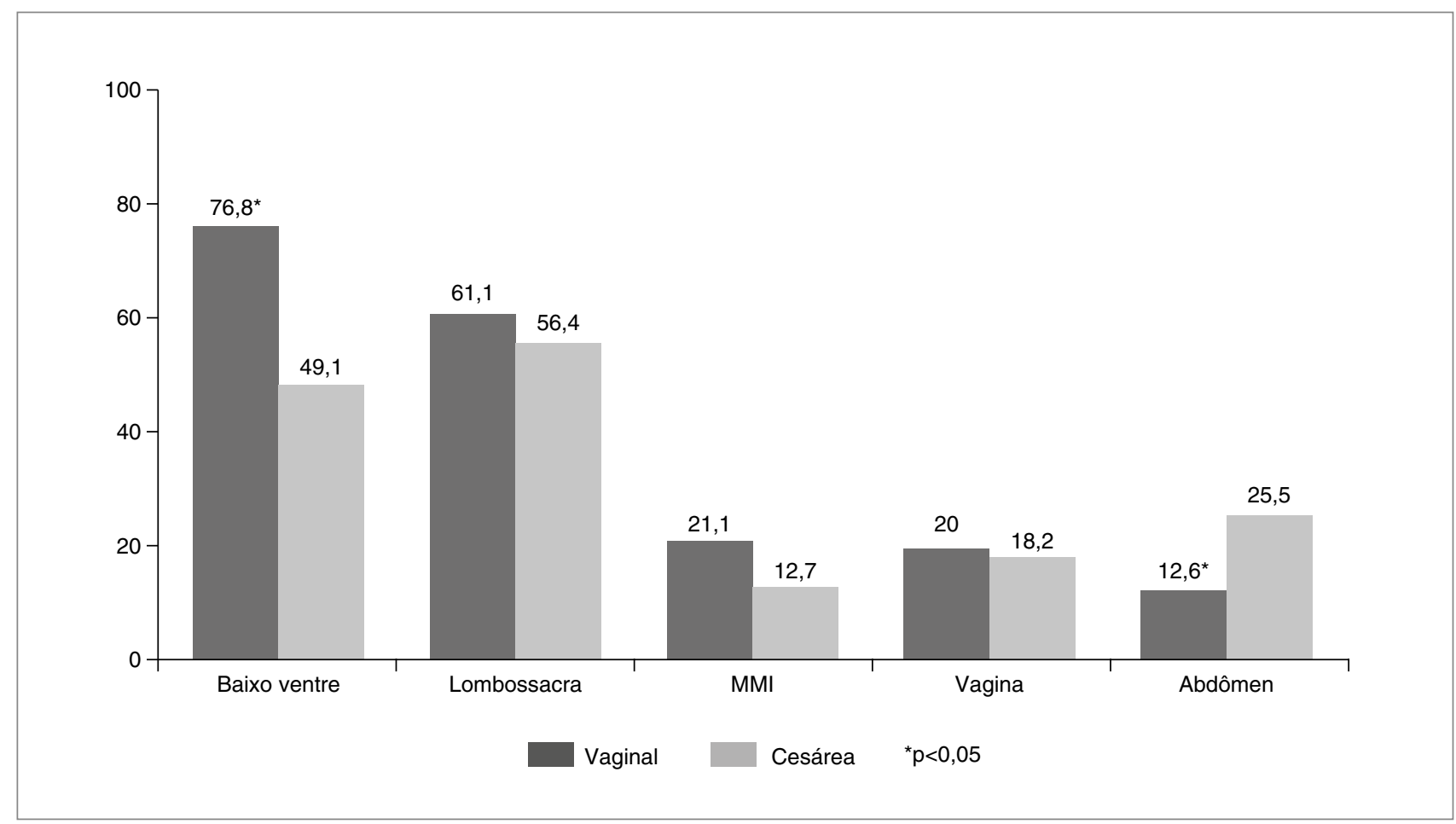

Figura 2: Localização da dor durante o trabalho de parto vaginal $(n=95)$ e cesariana $(n=55)$ 
escolha de via de parto neste estudo, não são possíveis inferências a respeito desta variável.

Mas, em relação ao PP, a experiência de dor é mais presente naquelas após cesariana ${ }^{10}$ apresentando maior frequência de pontos dolorosos e maior intensificação no IDA. Contudo, diversos estudos apresentam percepções positivas e negativas das mulheres sobre os dois tipos de parto ${ }^{8}$.

Os descritores mais frequentes para caracterizar a dor foram relacionados tanto a sensações físicas (aguda, dolorida, cólica e cansativa-exaustiva) quanto a sensações emocionais (cruel-punitiva e amedrontadora). Contrariamente ao estudo realizado por Capogna et al. ${ }^{12}$, os descritores mais citados $(\geq 50 \%)$ foram ligados a sensações físicas: "cólica, queimação, pressionante, fincada, pesada, dolorida, cansativa-exaustiva e insuportável”, sendo que o questionário foi aplicado no TP, o que sugere a diferença na escolha dos descritores. Souza et al. ${ }^{10}$ encontraram relatos de dor que "incomoda", "que prende" e "que repuxa" exclusivamente após a cesariana, utilizando o questionário de McGill em sua forma completa.

Os pontos dolorosos mais frequentes ( $\geq 20 \%)$ e comuns a ambas as vias de parto durante o TP foram, no geral, baixo ventre e lombossacra, propondo que os maiores geradores de dor durante o TP sejam a dilatação do colo uterino e o aumento da pressão sob as estruturas pélvicas e plexo lombossacro.

Os cuidados prestados, o acolhimento e práticas humanizadas são de extrema importância para o sucesso e satisfação desde o $\mathrm{PN}$ até o parto ${ }^{6-9}$. As maternidades públicas têm se esforçado para adequar as políticas de saúde em seu cotidiano9 ${ }^{9}$, mas mesmo assim, o SUS pode melhorar sua assistência e ainda tem lacunas a serem preenchidas.

Em estudo anterior ${ }^{18}$, ficou explícito que a resolução dos problemas de cuidados PN e de assistência ao parto não está centrada em avanços estruturais ou em sofisticação tecnológica, mas sim no comportamento e atitudes dos trabalhadores da saúde que, efetivamente, contribuam para o fortalecimento do vínculo entre profissionais, usuárias, família e comunidade. Todos os atores envolvidos na assistência obstétrica devem compreender as peculiaridades desse momento, a vulnerabilidade materna, respeitar os valores e culturas e prestar uma assistência qualificada e humana.

Diante disso, reafirma-se a necessidade de adequação da assistência ao parto aos programas propostos pelo Ministério da Saúde, para garantir atendimento e acompanhamento adequados desde o PN até o puerpério. Os cuidados prestados, o acolhimento e práticas humanizadas são de extrema importância para o sucesso e satisfação desde o $\mathrm{PN}$ até o parto ${ }^{6-9}$. As maternidades públicas têm se esforçado para adequar as políticas de saúde em seu cotidiano ${ }^{9}$, mas mesmo assim, o SUS pode melhorar sua assistência e ainda tem lacunas a serem preenchidas. Destaca-se uma necessidade imperiosa de mudança em diversos aspectos de atendimento e de educação dos profissionais de saúde associada à melhoria de condições de trabalho que em sua maioria são precárias ${ }^{3}$.

Embora seja um assunto bastante estudado e discutido, a dor ainda se apresenta como um fator de influência negativa na satisfação e escolha da via de parto e, portanto, os profissionais devem perceber a necessidade de se investir em técnicas de manejo da dor, farmacológicas ou não-farmacológicas, para melhor satisfação das mulheres com relação à experiência do parto, assim como orientá-las em todas as etapas. Espera-se que este estudo contribua para futuras pesquisas e práticas clínicas relacionadas à assistência, manejo da dor e satisfação durante o parto, para que a mulher possa atuar como participante ativa deste processo.

\section{AGRADECIMENTOS}

Às mulheres que participaram deste estudo e às equipes das maternidades Nossa Senhora de Lourdes e Hospital Santa Isabel.

\section{REFERÊNCIAS}

1. Brasil. Ministério da Saúde. Pré-natal e puerpério: atenção qualificada e humanizada. Manual técnico. Brasília: Ministério da Saúde; 2006

2. Serruya SJ, Ceccati JG, Lago TG. O Programa de humanização no pré-natal e nascimento do Ministério da Saúde no Brasil: resultados iniciais. Cad Saúde Pública. 2004;20(5):1281-9. http://dx.doi.org/10.1590/S0102-311X2004000500022

3. Fernandes CE. Três percepções sobre gestação e puerpério ABCS Health Sci. 2015;40(2):60-1.

http://dx.doi.org/10.7322/abcshs.v40i2.731

4. Rodrigues AV, Siqueira AAF. Sobre as dores e temores do parto: dimensões de uma escuta. Rev Bras Saúde Mater Infant. 2008;8(2):179-86.

http://dx.doi.org/10.1590/S1519-38292008000200005
5. D'orsi E, Brüggemann OM, Diniz CSG, Aguiar JM, Gusman CR, Torres JA, et al. Desigualdades sociais e satisfação das mulheres com o atendimento ao parto no Brasil: estudo nacional de base hospitalar. Cad Saúde Pública. 2014;30(Suppl. 1):S154-68

http://dx.doi.org/10.1590/0102-311X00087813

6. Pereira RR, Franco SC, Baldin N. Representações sociais e decisões das gestantes sobre a parturição: protagonismo das mulheres. Saúde Soc. 2011;20(3):579-89. http://dx.doi.org/10.1590/S0104-12902011000300005

7. Gama AS, Giffin KM, Angulo-Tuesta A, Barbosa GP, D'orsi E. Representações e experiências das mulheres sobre a assistência ao parto vaginal e cesárea em maternidades pública e privada. Cad Saúde Pública. 2009;25(11):2480-8.

http://dx.doi.org/10.1590/S0102-311X2009001100017 
8. Velho MB, Santos EKA, Brüggemann OM, Camargo BV. Vivência do parto normal ou cesáreo: revisão integrativa sobre a percepção de mulheres. Texto Contexto Enferm. 2012;21(2):458-66. http://dx.doi.org/10.1590/S0104-07072012000200026

9. Vieira SM, Bock LF, Zocche DA, Pessota CU. Percepção das puérperas sobre a assistência prestada pela equipe de saúde no pré-natal. Texto Contexto Enferm. 2011;20(spe):255-62. http://dx.doi.org/10.1590/S0104-07072011000500032

10. Souza L, Pitangui ACR, Gomes FA, Nakano AMS, Ferreira CHJ. Mensuração e características de dor após cesárea e sua relação com limitação de atividades. Acta Paul Enferm. 2009;22(6):741-47.

11. Beleza ACS, Ferreira CHJ, Souza L, Nakano AMS. Mensuração e caracterização da dor após episiotomia e sua relação com a limitação de atividades. Rev Bras Enferm. 2012;65(2):264-8. http://dx.doi.org/10.1590/S0034-71672012000200010

12. Capogna G, Camorcia M, Stirparo M, Valentini G, Grassiano A, Farcomeni A. Multidimensional evaluation of pain during early and late labor: a comparison of nulliparous and multiparous women. Inter J Obstet Anest. 2010;19(2):167-70. http://dx.doi.org/10.1016/j.ijoa.2009.05.013

13. Santana LS, Gallo RBS, Ferreira CHJ, Quintana SM, Marcolin AC. Pain location during early active labor stage. Rev Dor. 2013;14(3):184-6. http://dx.doi.org/10.1590/S1806-00132013000300006
14. Domingues RMSM, Dias MAB, Nakamura-Pereira M, Torres JA d'Orsi E, Pereira APE, et al. Processo de decisão pelo tipo de parto no Brasil: da preferência inicial das mulheres à via de parto final. Cad Saúde Pública. 2014;30(Suppl. 1):S101-16. http://dx.doi.org/10.1590/0102-311X00105113

15. Costa R, Figueiredo B, Pacheco A, Pais A. Parto: expectativas, experiências, dor e satisfação. Psicol Saúde Doenças. 2003:4(1):47-67.

16. Gurgel RQ, Nery AMDG, Almeida MLD, Oliveira ERR, Lima DFD, Bettiol $\mathrm{H}$, et al. Características das gestações, partos e recémnascidos da região metropolitana de Aracaju, Sergipe, Brasil. Rev Bras Saúde Matern Infant. 2009;9(2):167-77. http://dx.doi.org/10.1590/S1519-38292009000200006

17. Menezes Costa LC, Maher CG, Mcauley JH, Hancock MJ, Melo Oliveira W, Azevedo DC, et al. The Brazilian-Portuguese versions of the McGill Pain Questionnaire were reproducible, valid, and responsive in patients with musculoskeletal pain. J Clin Epidemiol. 2011;64(8):903-12. http://dx.doi.org/10.1016/j.jclinepi.2010.12.009

18. Luz NF, Assis TR, Rezende FR. Puérperas adolescentes: percepções relacionadas ao pré-natal e ao parto. ABCS Health Sci. 2015;40(2):80-4.

http://dx.doi.org/10.7322/abcshs.v40i2.735 